\title{
Ground Influence on the Input Impedance of Transient Dipole and Bow-Tie Antennas
}

\author{
Andrian Andaya Lestari, Alexander G. Yarovoy, Member, IEEE, and Leo P. Ligthart, Fellow, IEEE
}

\begin{abstract}
In this paper, the influence of a lossy ground on the input impedance of dipole and bow-tie antennas excited by a short pulse is investigated. It is shown that the ground influence on the input impedance of transient dipole and bow-tie antennas is significant only for elevations smaller than $1 / 5$ of the wavelength that corresponds to the central frequency of the exciting pulse. Furthermore, a principal difference between the input impedance due to traveling-wave and standing-wave current distributions is pointed out.
\end{abstract}

Index Terms-Bow-tie antenna, dipole antenna, input impedance, transient antenna.

\section{INTRODUCTION}

D IPOLE and bow-tie antennas are employed in many transient applications such as impulse ground penetrating radar (GPR) for transmitting short transient pulses. The large antenna bandwidth required to transmit such pulses with minimal distortion (e.g., antenna ringing) is usually obtained by the application of resistive loading [1], [2]. As resistive loading substantially reduces radiation efficiency [1], it is essential to achieve maximum power transfer from the generator to the antenna, for which the input impedance of the antenna should be known.

The input impedances of time-harmonic and transient antennas are principally different since the former is due to standing-wave current distribution, while the latter is due to traveling-wave current distribution. Publications with regard to the input impedance of time-harmonic dipole and bow-tie antennas near the ground are abundantly available in the literature. On the contrary, not much of the input impedance of transient dipole and bow-tie antennas near the ground has been reported. In the free-space case, significant contributions were given by Wu [3] and Carrel [4] who presented analytical expressions of the input impedance of transient dipole and bow-tie antennas, respectively. In this paper we analyze the input impedance of transient dipole and bow-tie antennas near a lossy ground.

A numerical method to predict the input impedance of arbitrary metallic transient antennas in free space using the time-domain integral equation (TDIE) method has been demonstrated by Booker, et al. [5]. In their work the TDIE is

Manuscript received March 7, 2003; revised August 20, 2003. This work was supported by the Dutch Technology Foundation (STW) under the projects "Improved Ground Penetrating Radar Technology" (1999-2000) and "Advanced Re-Locatable Multisensor System for Buried Landmine Detection" (2001-2002).

The authors are with the International Research Centre for Telecommunications-Transmission and Radar (IRCTR), Delft University of Technology, Delft, The Netherlands (e-mail: a.lestari@irctr.tudelft.nl).

Digital Object Identifier 10.1109/TAP.2004.832371 numerically solved by the method of moments (MoM) using the marching-on-in time approach. By neglecting end reflections in the time-domain solution, the input impedance is given by the high-frequency limit of the frequency-domain solution obtained by Fourier transforming the mentioned time-domain solution. Unfortunately, when it comes to layered-medium problems, well-suited Green's functions in space-time domain are not yet well documented. One of few developments of such Green's functions has recently been reported for analyzing the response of a transient dipole in stratified media [6]. However, layered-medium Green's function formulations in space-time domain which are directly applicable to surface-patch MoM methodologies, are not yet widely reported.

The numerical analysis carried out in this work is based on the frequency-domain integral equation (FDIE) method, as the methods of solution for problems with layered media in frequency domain are already well established. The FDIE incorporates a layered-medium Green's function and is numerically solved by a surface-patch MoM scheme for metallic nonwire structures, whereas wire structures are approximated by narrow strips. The input impedance of the transient antennas is obtained by the Fourier transformation and a time-window technique for excluding end reflections. An experimental analysis is performed to verify the computed results.

\section{NUMERICAL METHOD}

The work reported in this paper is based on a frequencydomain mixed-potential integral equation (MPIE) formulation. To account for the presence of the ground the dyadic Green's function formulation $\mathrm{C}$ for layered-medium problems by Michalski and Zheng [7] is incorporated into the MPIE, which is numerically solved by MoM according to the triangular surface-patch methodology introduced by Rao, et al. [8]. Computation time is minimized by employing the efficient numerical implementation introduced in [9].

In this work the antennas are excited by a monocycle with 0.8-ns duration shown in Fig. 1(a). In Fig. 1(b) its normalized spectrum is given. The central frequency of this pulse is about $1 \mathrm{GHz}$ and the $-3 \mathrm{~dB}$ levels are found at frequencies $424 \mathrm{MHz}$ and $1.670 \mathrm{GHz}$. It can be seen in Fig. 1(b) that the spectrum of the exciting pulse is essentially contained within the $0-5 \mathrm{GHz}$ range.

In this paper, we develop a nonstraightforward numerical method to predict the input impedance of a transient antenna in four steps as follows.

1) Antenna feed current $J(\omega)$ is computed in the frequency domain with 1 Volt input voltage by means of the MoM 


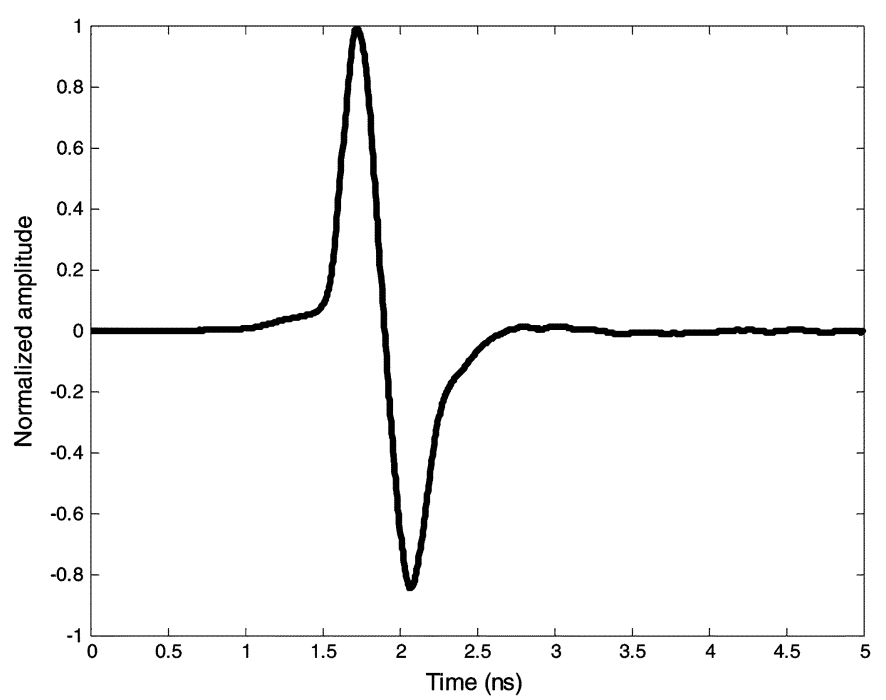

(a)

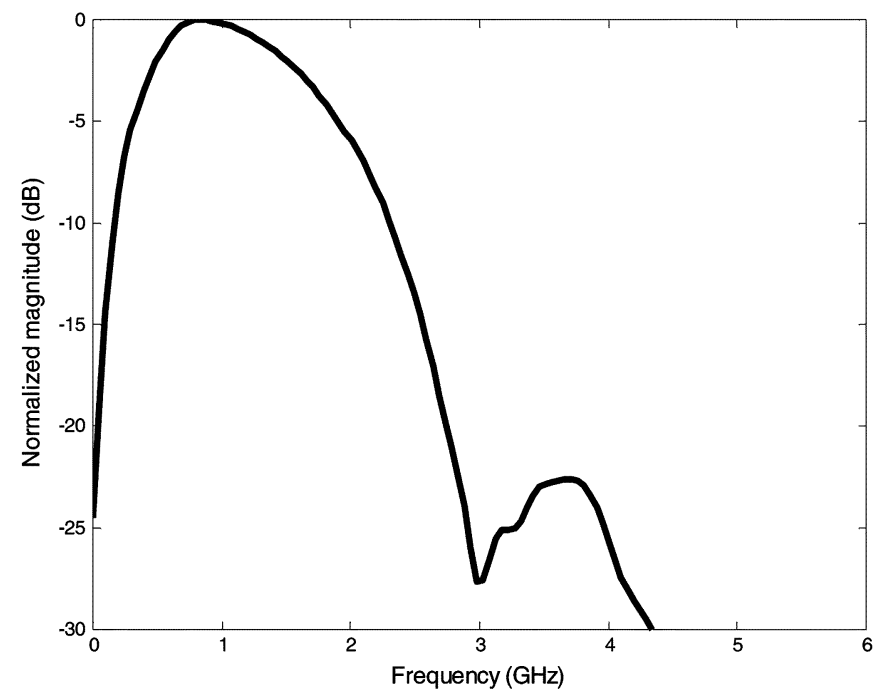

(b)

Fig. 1. Exciting pulse used in this work: (a) a monocycle with 0.8-ns duration and (b) its normalized spectrum.

scheme mentioned above. For obtaining time-domain solutions, the computations are performed at 100 frequency points from $50 \mathrm{MHz}$ to $5 \mathrm{GHz}$ with $50-\mathrm{MHz}$ steps.

2) Exciting pulse function $V(\omega)$ is synthesized directly from measurement of the 0.8-ns monocycle in Fig. 1(a), and used as the excitation model in the computations.

3) The feed current of the same antenna with infinite length (thus, no end reflections) is computed by means of the Fourier transformation and a time-window technique for removal of end reflections, which can concisely be written as

$$
J_{\infty}(\omega)=\mathrm{F}\left\{\mathrm{W}\left\{\mathrm{F}^{-1}\left[J(\omega) V_{\mathrm{N}}(\omega)\right]\right\}\right\}
$$

where $V_{\mathrm{N}}(\omega)$ is the normalized $V(\omega), \mathrm{F}$ is the discrete Fourier transformation operator, $\mathrm{F}^{-1}$ is the discrete inverse Fourier transformation operator, and $\mathrm{W}$ is the time-window operator with smooth truncation process given by

$$
\begin{aligned}
& A_{k}=w A_{k+1}, \quad k=-1,-2 \ldots \quad \text { for the left end } \\
& A_{k}=w A_{k-1}, \quad k=1,2 \ldots \quad \text { for the right end }
\end{aligned}
$$

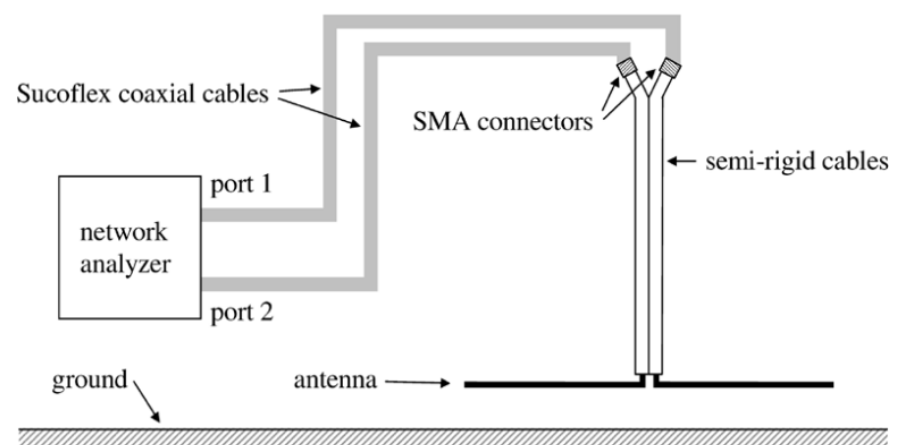

Fig. 2. Setup for input impedance measurements.

in which $A$ is the value of the real or imaginary part of the argument, and $w$ is the decay rate of the truncation process that assumes values in the range $0 \leq w<1$. We have found that typically the performance of (2) is optimal with $w=$ 0.7 . Note that when $w=0$ (2) reduces to a rectangular time window.

4) Finally, the input impedance of the transient antenna is obtained as

$$
Z_{0}^{\mathrm{ant}}(\omega)=\frac{V_{\mathrm{N}}(\omega)}{J_{\infty}(\omega)}
$$

\section{Measurement Technique}

We perform input impedance measurements in frequency domain using a standard network analyzer. The antennas are situated horizontally over a lossy ground, which in this case is dry sand. The measurements are carried out without an anechoic chamber, and consequently the results are heavily disturbed by reflections from surrounding objects. To deal with this, the measurement results are inverse-Fourier transformed into time domain, after which use of time gating is made to remove those unwanted reflections. The actual impedance of the antenna can be extracted afterwards by performing the Fourier transformation of the results back to frequency domain.

Furthermore, to properly measure balanced loads such as the antennas discussed here, a balun is required. However, since ultra-wideband baluns are difficult to produce and commercially available ones are expensive, baluns are not used in the measurements. A technique to measure the input impedance of balanced antennas without baluns introduced in [10] is here simplified. The antenna under test is fed using two identical 50-Ohm semi-rigid cables soldered together over their length, except for a small part near the ends where SMA connectors are attached. Each of the inner conductors of the other ends is soldered to one of the antenna terminals in the way shown in Fig. 2. The semi-rigid cables are connected by $50-\mathrm{Ohm}$ Sucoflex coaxial cables to the ports of the network analyzer, which has been previously calibrated at the SMA connectors. Hence, the reflection coefficient at the SMA connectors is given by

$$
\Gamma_{\mathrm{SMA}}=S_{11}-S_{12}
$$

where $S_{11}$ and $S_{12}$ are the $S$-parameters measured by the network analyzer. Disturbances caused by unwanted reflections can 
be removed from (4) by means of a time-gating operation, which can be described by

$$
\Gamma_{\mathrm{SMA}}^{\prime}=\mathrm{F}\left\{\mathrm{W}\left\{\mathrm{F}^{-1}\left[\Gamma_{\mathrm{SMA}}\right]\right\}\right\}
$$

in which the time-window operator $\mathrm{W}$ implements the truncation process in (2). Using the dielectric constant $\varepsilon_{\text {src }}$ and the insertion loss $L$ of the semi-rigid cables provided by the manufacturer, the reflection coefficient at the antenna terminal can then be written as

$$
\Gamma_{\mathrm{ant}}=\Gamma_{\mathrm{SMA}}^{\prime} e^{j \beta l} 10^{L / 20}
$$

where $\beta$ is the phase constant which depends on $\varepsilon_{\mathrm{src}}, l$ is two times the length of the semi-rigid cables and $L$ is given in $\mathrm{dB} / \mathrm{m}$. Several available values of $L$ for different frequencies are interpolated to obtain the values of $L$ over the whole frequency range. Finally, the input impedance of the antenna is given by

$$
Z_{\text {in }}=\frac{1+\Gamma_{\text {ant }}}{1-\Gamma_{\text {ant }}} Z_{0}^{\text {src }}
$$

where $Z_{0}^{\text {src }}$ is the characteristic impedance of the semi-rigid cables, which has a value of $100 \mathrm{Ohms}$ due to the double-line configuration shown in Fig. 2.

It is worth noting that antenna length is actually not important for the input impedance of transient antennas. However, in the above technique antenna length is important for computation and measurement procedures, i.e.,

- to ensure separation in time between feed-point and end reflections for time window application,

- to obtain adequate duration of the time window since a finite time window limits the lowest frequency at which input impedance can be determined.

In this work we use antennas with 50-cm length, which allows application of a time window with 2-ns duration for excitation with the 0.8 -ns monocycle.

\section{ANALYSIS}

As the input impedance of a transient dipole is frequency dependent [3], the input impedance is determined with respect to the exciting pulse using the averaging given by [11]

$$
Z_{\mathrm{ave}}=\frac{1}{\omega_{2}-\omega_{1}} \int_{\omega_{1}}^{\omega_{2}} V_{\mathrm{N}}(\omega) Z(\omega) d \omega
$$

in which $\omega_{1}$ and $\omega_{2}$ are the frequencies which correspond to the lower and upper limits of the pulse spectrum, respectively, and $Z(\omega)$ is the antenna input impedance. It has been indicated in [11] that it is adequate to assume $V_{\mathrm{N}}(\omega)=1$ over the range $\omega_{1} \leq \omega \leq \omega_{2}$, and this is followed here with $\omega_{1}$ and $\omega_{2}$ corresponding to the $-3 \mathrm{~dB}$ limits of the exciting pulse in Fig. 1. Hence, $Z_{\text {ave }}$ in (8) can be interpreted as the input impedance with respect to the exciting pulse (the 0.8 -ns monocycle). In this work $Z(\omega)$ in (8) is replaced with $Z_{0}^{\text {ant }}(\omega)$ in (3). In this way, (8) is improved because prior to using the averaging in (8) we

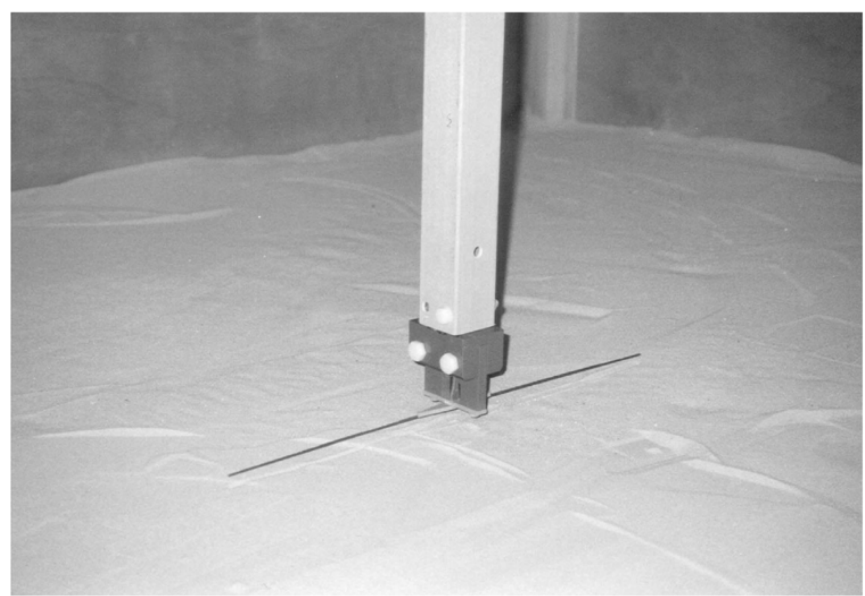

Fig. 3. Experimental dipole over dry sand. Length $=50 \mathrm{~cm}$ and wire diameter $=2 \mathrm{~mm}$.

perform time windowing in (1), which greatly reduces the oscillation of the impedance curve. In effect, this approach improves the averaging process.

The computed and measured input impedances of the horizontal transient dipole in Fig. 3 with respect to the 0.8-ns monocycle as function of elevation above the sand are presented in Fig. 4(a). The wire is modeled as a thin strip using the equivalent radius formula [12]. The result computed by the Numerical Electromagnetics Code (NEC-2) is included for comparison. It can be seen that in general the computations agree with the measurement. At the highest elevation the computed and measured values of the reactance are about $200 \Omega$, in accordance with the result obtained using the expression given in [3]. For very small distances from the interface we observe that the result computed by the triangular-patch MoM suffers from a slight discrepancy with the measurement. We notice that this discrepancy might be caused by the variation of the electrical length of the feed gap of the experimental antenna when it approaches the ground. Such a phenomenon is however not experienced by the delta-function generator assumed in the triangular-patch MoM. The delta-gap model used by NEC, on the other hand, accommodates this phenomenon as it uses a feed segment with the same length as the feed gap width of the experimental antenna. This leads to better agreement with the measurement for small distances from the interface as shown in the figure. The result computed using the commercial MoM code FEKO, which is based on the same triangular-patch MoM methodology, is also included. It can be seen that generally agreement between our code and FEKO is achieved. To further test the accuracy of our results, computations are carried out using our code and FEKO for a 1-mm wire diameter and the results are presented in Fig. 4(b), in which the results for a 2-mm diameter in Fig. 4(a) are also shown. It is demonstrated in Fig. 4(b) that agreement is generally achieved. The observed slight discrepancy in reactance may be attributed to different densities of the mesh generated by the codes, especially in the feed region of the antenna. Moreover, we note that agreement between the results for small elevations indicates the accuracy of numerical evaluation of the layered-medium Green's function. 


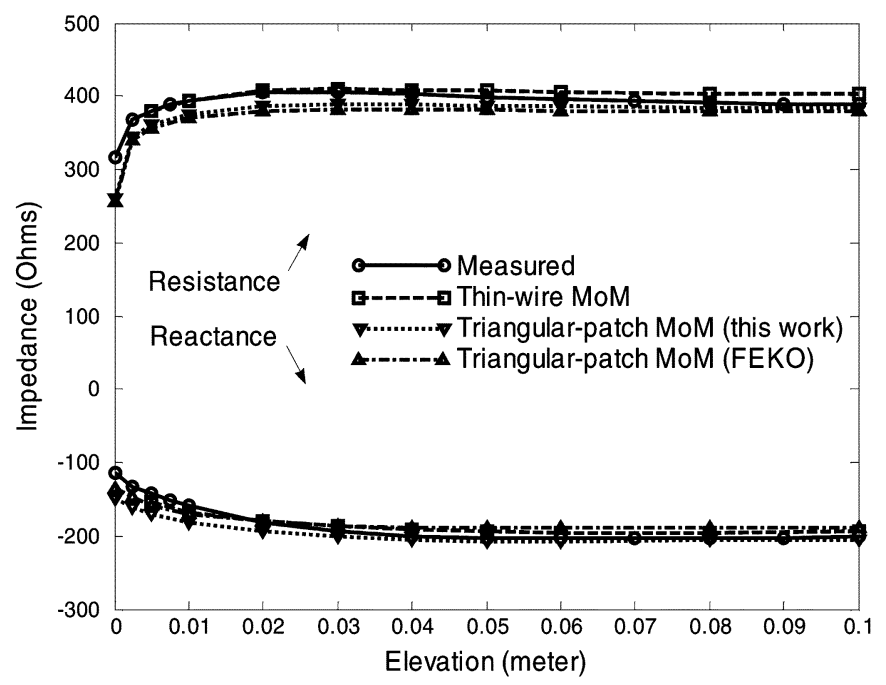

(a)

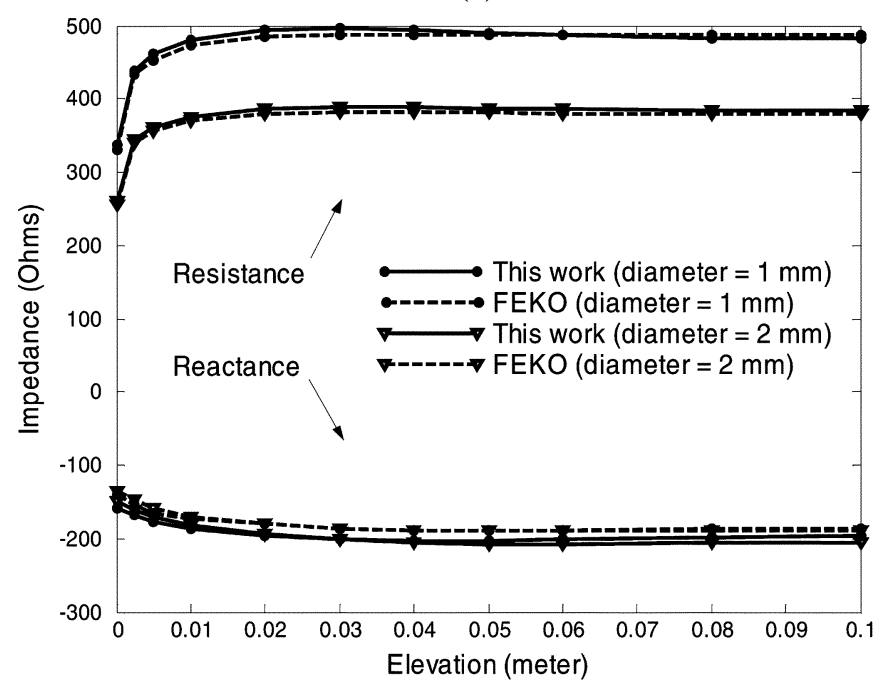

(b)

Fig. 4. (a) Input impedance of the transient dipole in Fig. 3 with respect to the 0.8 -ns monocycle as function of elevation above the sand. (b) Comparison between this work and FEKO for two different wire diameters (1 and $2 \mathrm{~mm}$ ).

Additional proof of the accuracy of the numerical results is given in Fig. 5 by comparison with analytical results, obtained using the theory in [3]. The input impedance of a transient dipole in free space is plotted for two different wire diameters, 1 and 2 $\mathrm{mm}$. It can be seen that for sufficiently high frequencies generally agreement between numerical and analytical results is achieved. The observed slight discrepancy at high frequencies may be explained by difference in the excitation models of the antenna. The theory in [3] assumes excitation from a coaxial feed system, while for excitation we use a delta-function generator. The large discrepancy at low frequencies is due to the finite length of the time window, which imposes limitation on the lowest frequency at which accuracy of the result is ensured. To obtain improved accuracy at lower frequencies one should use a longer antenna for allowing a longer time window.

It is advisable to mention the advantages and disadvantages of the used codes. The advantage of NEC-2 is the efficiency for handling wire structures as it employs thin-wire approximation, which reduces formulation of the problem into a

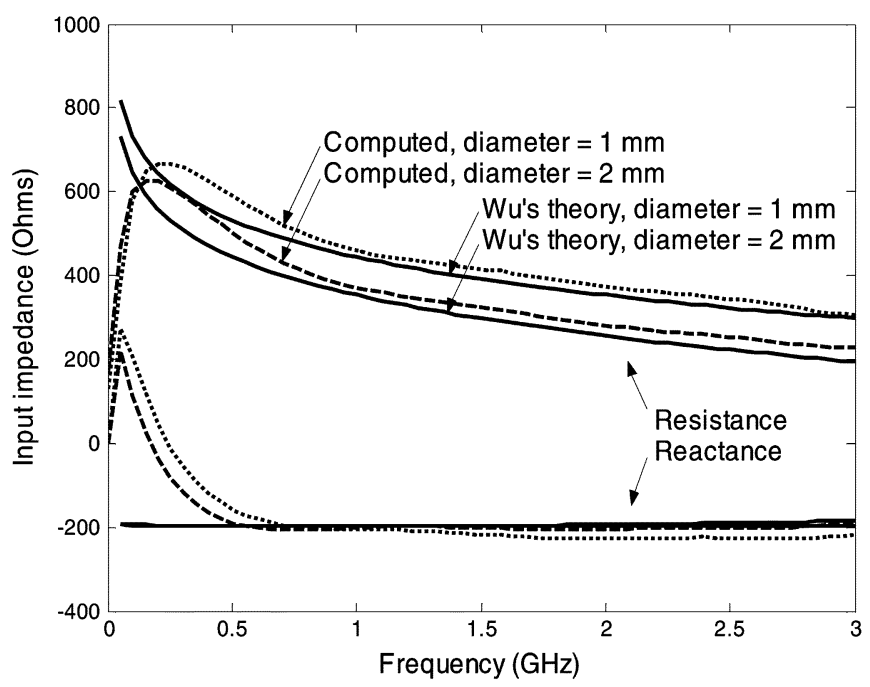

Fig. 5. Input impedance of transient dipoles in free space for two different wire diameters: analytical against numerical results. Analytical results are obtained using [3]; numerical results are computed by our code.

one-dimensional integral equation. However, to this work the main drawback of NEC-2 is that it renders inaccurate when modeling antennas very close to the ground. In Fig. 4(a) NEC-2 computation is interrupted at $5-\mathrm{mm}$ elevation because for lower elevations the results become inaccurate. One of the advantages of our triangular-patch MoM code is its capability of modeling antennas touching the interface. In addition, it offers flexibility for modeling metallic antennas of arbitrary shape. In comparison with NEC-2 the obvious disadvantage of the code when treating wire structures is its lower computation efficiency since it employs a surface integral formulation.

To analyze the influence of a lossy ground on the input impedance of the transient dipole we compute the input impedance with respect to the 0.8 -ns monocycle as functions of antenna elevation for different ground types. In particular, we assume the ground to be sandy soil $\left(\varepsilon_{\mathrm{r}}=4, \sigma=0.004 \mathrm{~S} / \mathrm{m}\right)$, dry clay $\left(\varepsilon_{\mathrm{r}}=16, \sigma=0.03 \mathrm{~S} / \mathrm{m}\right)$, wet clay $\left(\varepsilon_{\mathrm{r}}=25, \sigma=0.06 \mathrm{~S} / \mathrm{m}\right)$, and muddy soil $\left(\varepsilon_{\mathrm{r}}=50, \sigma=0.2 \mathrm{~S} / \mathrm{m}\right)$. Furthermore, two different wire diameters of 1 and $2 \mathrm{~mm}$ are assumed to investigate the influence of the wire thickness on the results. The computed input resistance and reactance with respect to the 0.8 -ns monocycle are plotted in Fig. 6, where it is evident that the presence of the ground significantly affects the impedance only for very small distances from the interface. In Fig. 6 it is shown that, the ground influence is already very small at elevations higher than $6 \mathrm{~cm}$ for a wide range of ground types. Noting that the central frequency of the $0.8-\mathrm{ns}$ monocycle is about $1 \mathrm{GHz}$ (corresponding to a wavelength of $30 \mathrm{~cm}$ ), as a generalization of the results one may state that the ground essentially affects the input impedance of a transient dipole only when the antenna elevation is smaller than $1 / 5$ of the wavelength that corresponds to the central frequency of the exciting pulse.

Evidently, wire diameter exhibits a considerable influence on the resistance as demonstrated in Fig. 6, which indicates that doubling the wire diameter from 1 to $2 \mathrm{~mm}$ reduces the resistance by about $19 \%$. It is also worth noting that for a very 

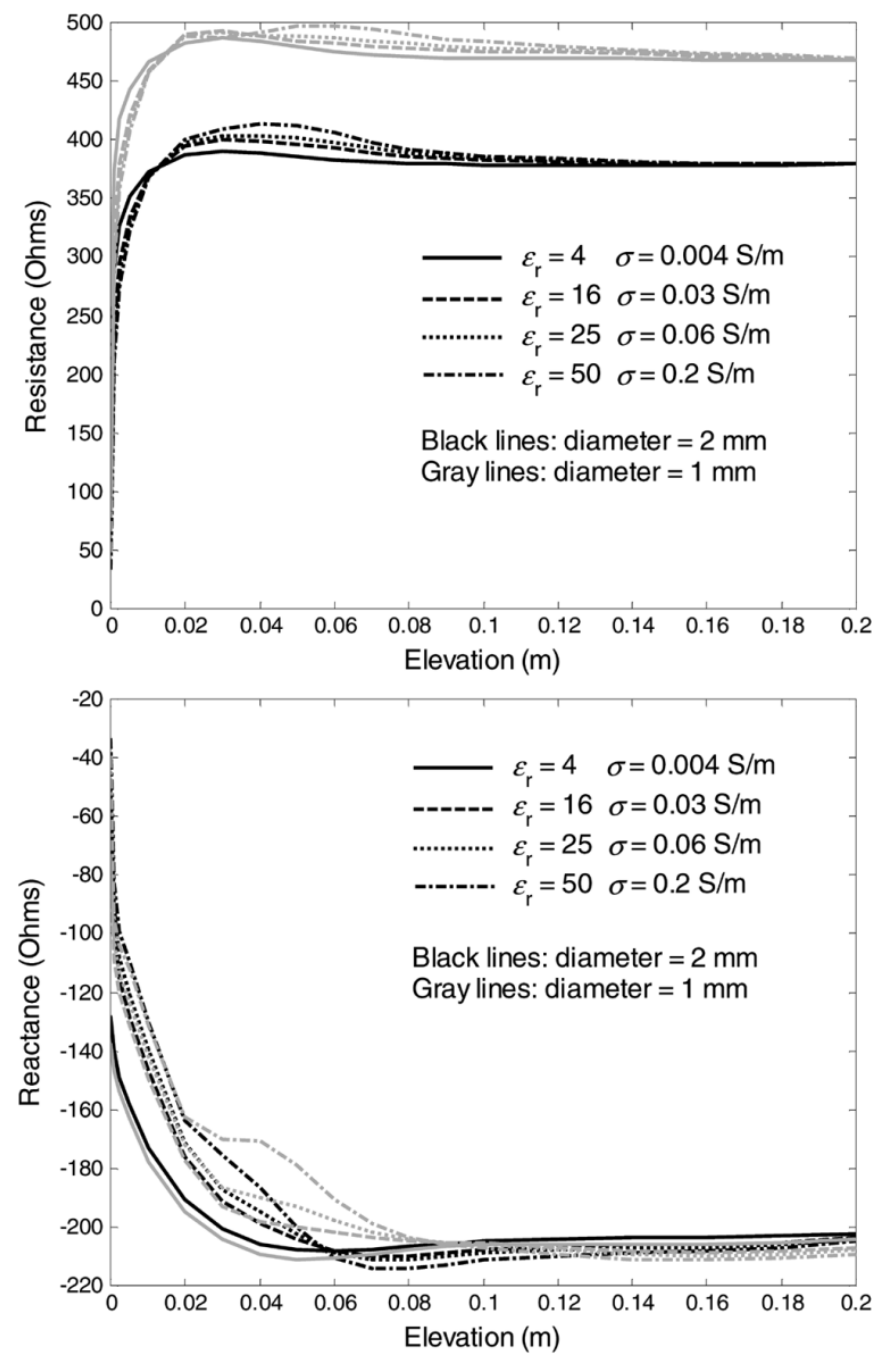

Fig. 6. Computed input impedance of a transient dipole with respect to the 0.8 -ns monocycle as functions of elevation for different ground types.

close proximity to the interface, the input resistance decreases as the dipole approaches the interface. We note that this behavior is the opposite of the time-harmonic case, in which for very small distances from the interface the input resistance increases as the dipole is lowered [13], [14]. This result indicates a principal difference between traveling-wave and standing-wave current distributions of transient and time-harmonic antennas, respectively.

In this paper the input impedance of a transient bow-tie antenna is computed as functions of elevation above the ground for flare angles of $30^{\circ}, 50^{\circ}$, and $70^{\circ}$. The computed input impedance of a transient bow tie above sandy soil $\left(\varepsilon_{\mathrm{r}}=4\right.$ and $\sigma=0.004 \mathrm{~S} / \mathrm{m}$ ) with respect to the 0.8 -ns monocycle is presented in Fig. 7. We notice in the figure that for the three flare angles the value of the reactance at a $6-\mathrm{cm}$ elevation is already close to zero, which is the free-space value of the characteristic reactance of a transient bow tie [4]. Moreover, by inspection of the result given in [4] it is found that that at a $6-\mathrm{cm}$ elevation the resistance nearly assumes its free-space value. Hence, similar to the case of the transient dipole discussed previously it is shown that the ground essentially affects the input impedance of a transient bow-tie antenna only when the antenna elevation

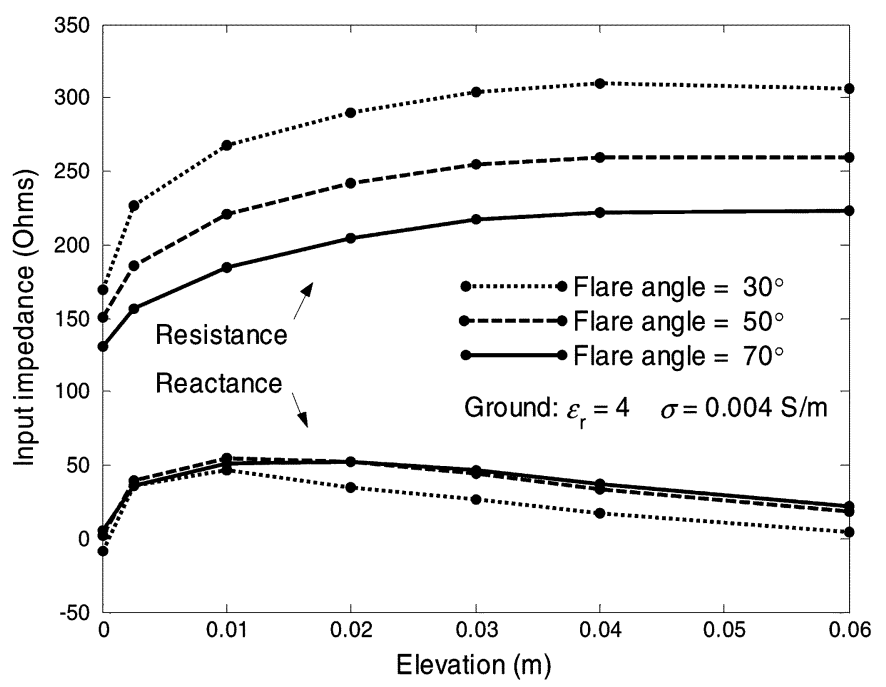

Fig. 7. Computed input impedance of a transient bow tie as functions of elevation above sandy soil for different flare angles.

is smaller than $1 / 5$ of the wavelength that corresponds to the central frequency of the exciting pulse.

\section{CONCLUSION}

The influence of a lossy ground on the input impedance of dipole and bow-tie antennas excited by a short pulse is investigated. It is shown that the ground influence on the input impedance of a transient dipole and bow-tie antennas is significant only for elevations smaller than $1 / 5$ of the wavelength that corresponds to the central frequency of the exciting pulse. Furthermore, it is shown that for a very close proximity to the interface, the input resistance of a transient dipole decreases as the dipole approaches the interface. This behavior is the opposite of the time-harmonic case, in which for very small distances from the interface the input resistance increases as the dipole is lowered.

\section{ACKNOWLEDGMENT}

The authors thank P. Hakkaart for his assistance in the construction of the experimental antenna and J. Zijderveld for his assistance in the measurements.

\section{REFERENCES}

[1] T. P. Montoya and G. S. Smith, "A study of pulse radiation from several broad-band loaded monopoles," IEEE Trans. Antennas Propagat., vol. 44, pp. 1172-1182, Aug. 1996.

[2] K. L. Shlager, G. S. Smith, and J. G. Maloney, "Optimization of bow-tie antennas for pulse radiation," IEEE Trans. Antennas Propagat., vol. 42, pp. 975-982, July 1994.

[3] T. T. Wu, "Input admittance of infinitely long dipole antennas driven from coaxial lines," J. Math. Phys., vol. 3, pp. 1298-1301, 1962.

[4] R. L. Carrel, "The characteristic impedance of two infinite cones of arbitrary cross section," IRE Trans. Antennas Propagat., vol. AP-6, pp. 197-201, Apr. 1958.

[5] S. M. Booker, A. P. Lambert, and P. D. Smith, "A numerical calculation of transient antenna impedance," in Proc. 2nd Int. Conf. Computation in Electromagnetics, 1994, pp. 359-362.

[6] A. G. Tijhuis and A. Rubio Bretones, "Transient excitation of a layered dielectric medium by a pulsed electric dipole," IEEE Trans. Antennas Propagat., vol. 48, pp. 1673-1684, Oct. 2000. 
[7] K. A. Michalski and D. Zheng, "Electromagnetic scattering and radiation by surfaces of arbitrary shape in layered media, part I: Theory, part II: Implementation and results for contiguous half-spaces," IEEE Trans. Antennas Propagat., vol. 38, pp. 335-352, Mar. 1990.

[8] S. M. Rao, D. R. Wilton, and A. W. Glisson, "Electromagnetic scattering by surfaces of arbitrary shape," IEEE Trans. Antennas Propagat., vol. AP-30, pp. 409-418, May 1982.

[9] A. A. Lestari, A. G. Yarovoy, and L. P. Ligthart, "Numerical and experimental analysis of circular-end wire bow-tie antennas over a lossy ground," IEEE Trans. Antennas Propagat., vol. 52, pp. 26-35, Jan. 2004.

[10] K. D. Palmer and M. W. van Rooyen, "Simple broadband measurement of balanced loads using a network analyzer," in CD-ROM Proc. Millenium Conf. Antennas Propagat. (AP-2000), Davos, Switzerland, Apr. 2000.

[11] R. W. P. King and H. J. Schmitt, "The transient response of linear antennas and loops," IRE Trans. Antennas Propagat., vol. 10, pp. 222-228, May 1962.

[12] C. M. Butler, "The equivalent radius of a narrow conducting strip," IEEE Trans. Antennas Propagat., vol. AP-30, pp. 755-758, July 1982.

[13] G. Turner, "The Influence of subsurface properties on ground penetrating radar pulses," Ph.D. dissertation, Macquarie University, Sydney, NSW, Australia, 1993.

[14] C. J. Leat, N. V. Shuley, and G. F. Stickley, "Complex image model for ground-penetrating radar antennas," IEEE Trans. Antennas Propagat., vol. 46, pp. 1483-1488, Oct. 1998.

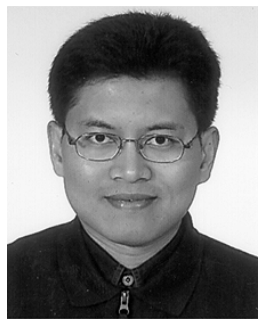

Andrian Andaya Lestari was born in Bogor, Indonesia. He received the Ingenieur and Ph.D. degrees in electrical engineering from Delft University of Technology, Delft, The Netherlands, in 1993 and 2003, respectively.

From 1993 to 1998 , he was with a government research agency in Jakarta, Indonesia. He joined the International Research Centre for Telecommunications-transmission and Radar (IRCTR), Delft University of Technology, as a Researcher in 1998. His work at IRCTR has resulted in over 20 publications, which include national and international patents, journal and conference papers, and scientific reports. Currently he works on ultrawide-band antennas and numerical tools for transient antenna analysis.

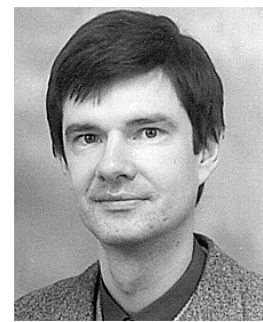

Alexander G. Yarovoy (M'96) received the Diploma (with honors) in radiophysics and electronics and the Cand. Phys. \& Math. Sci. and Dr. Phys. \& Math. Sci. degrees in radiophysics, from Kharkov State University, Kharkov, Ukraine, in 1984, 1987, and 1994, respectively.

In 1987, he joined the Department of Radiophysics, Kharkov State University, as a Researcher and became a Professor in 1997. From September 1994 through 1996, he was with the Technical University of Ilmenau, Germany, as a Visiting Researcher. Since 1999, he has been with the International Research Centre for Telecommunications-Transmission and Radar (IRCTR), Delft University of Technology, Delft, The Netherlands, where he coordinates all GPR-related projects. His main research interests are in ultrawide-band electromagnetics, wave scattering from statistically rough surfaces and penetrable obstacles and computational methods in electromagnetics.

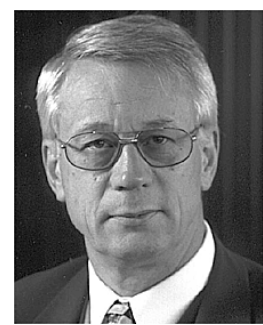

Leo P. Ligthart (M'94-SM'95-F'02) was born in Rotterdam, the Netherlands, on September 15, 1946. $\mathrm{He}$ received the Engineer's degree (cum laude) and the Doctor of Technology degree from Delf University of Technology, Delft, The Netherlands, in 1969 and 1985, respectively, the Doctorates (honoris causa) from Moscow State Technical University of Civil Aviation, Moscow, Russia, in 1999, and the Doctorates (honoris causa) from Tomsk State University of Control Systems and Radioelectronics, Tomsk, Russia, in 2001.

Since 1992, he has held the Chair of Microwave Transmission, Radar and Remote Sensing in the Department of Information Technology and Systems, Delft University of Technology, where in 1994, he became Director of the International Research Centre for Telecommunications-Transmission and Radar His principal areas of specialization include antennas and propagation, radar and remote sensing, but he has also been active in satellite, mobile, and radio communications. 